\title{
Sepsis neonatal tardía nosocomial en una unidad de terapia intensiva: agentes etiológicos y localización más frecuente
}

\author{
Gloria Celeste Samudio', Ruth Monzón', Lidia María Ortiz' y Gladys Maribel Godoy'
}

Late onset neonatal sepsis in an intensive care neonatal unit: etiological agents and most frequent location

Introduction: Nosocomial neonatal sepsis (NNS) is a frequent entity in intensive care units, causing great morbidity and mortality. The most frequent site is blood, followed by lungs and urine. Objective: To know the etiology and most frequent localization of infection in the NNS. Population, Material and Methods: Cross sectional study, from January to December 2015, performed in a teaching hospital. All newborns infants were included. Results: 70 patients were included, 88 episodes of NNS were analyzed. The most frequent localization was bacteremia in $40 \%$ of cases, followed by urinary tract infection and VAP in $25 \%$ respectively. The bacteria most frequently isolated were staphylococci of different types, followed by multiresistant Acinetobacter. The CNS involvement was $32 \%$. Mortality was $34 \%$, rising up to $50 \%$ with a second episode of NNS. The empirical therapy of choice was vancomycin and carbapenem, adjusting to antibiogram. Conclusions: The most frequent infection was bacteremia, mainly by staphylococci resistant to methicillin. CNS involvement was elevated, as well as mortality.

Keywords: Late onset neonatal sepsis, nosocomial neonatal sepsis, etiology, infection sites.

Palabras clave: Sepsis neonatal tardía, sepsis neonatal noscocomial, etiología, sitios de infección.

\section{Introducción}

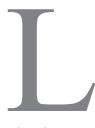

a sepsis nosocomial es una patología frecuente en las unidades de cuidados intensivos neonatales, en cuya etiopatogenia están involucrados múltiples factores, tales como el peso al nacimiento, edad gestacional, sexo, gravedad de la enfermedad, el uso de antimicrobianos, nutrición parenteral, los reiterados procedimientos invasores tales como catéteres venosos centrales, ventilación asistida y la inmadurez de ciertos órganos, a los que se suma la inmunodeficiencia propia del período neonatal ${ }^{1-3}$.

La prevalencia de esta patología varía en los diferentes centros, dependiendo del tipo de paciente, número y tipo de procedimientos invasores, estancia hospitalaria y prescripción de antimicrobianos, pudiendo llegar la frecuencia del uso de antimicrobianos hasta $84 \%$ en algunos centros ${ }^{4-7}$.

La sospecha de una infección nosocomial se establece luego de 48 a $72 \mathrm{~h}$ de ingresar a un hospital ${ }^{8}$ y se basa en la presencia de signos y síntomas clínicos y alteraciones de laboratorio compatibles con infección, tales como: presencia de leucocitosis o leucopenia, neutrofilia o neutropenia, relación elevada de leucocitos inmaduros/ neutrófilos totales, plaquetopenia y proteína $\mathrm{C}$ reactiva elevada. Todos ellos deben estar ausentes al ingreso hospitalario, o bien presentarse luego de la mejoría del cuadro que motivó el ingreso9.
Las cifras de mortalidad causadas por las infecciones nosocomiales en población neonatal son muy variables según los centros y países que los reporten ${ }^{6,10}$.

Los microoganismos implicados son diversos, siendo preocupante la presencia de bacterias altamente resistentes a múltiples antimicrobianos, entre ellos, cocáceas grampositivas del género Staphylococcus, especialmente las especies coagulasa negativas, bacterias gramnegativas tales como Pseudomonas aeruginosa y Klebsiella pneumoniae entre otros; además de hongos del tipo Candida sp. ${ }^{7,10-12}$. La documentación etiológica varía de 20 a $40 \%$ en sangre, con porcentajes variables en otros tipos de cultivos ${ }^{13-16}$.

Las infecciones nosocomiales más frecuentes en neonatos internados en unidades de cuidados neonatales son bacteriemias, neumonía asociada a ventilador mecánico (NAVM) e infección del tracto urinario (ITU); estas últimas pueden llegar a constituir hasta $10 \%$ de las infecciones nosocomiales ${ }^{13-15}$.

La mortalidad sigue siendo elevada, a pesar de las intervenciones realizadas en diversos centros ${ }^{6,12}$. El diagnóstico oportuno y tratamiento adecuado son imperativos para disminuir la mortalidad y morbilidad ocasionados por esta entidad. Es necesario que se realice una vigilancia activa de los microorganismos causantes de esta patología, especialmente de sus patrones de resistencia bacteriana, de modo a poder adecuar el tratamiento empírico inicial, conforme varíe la epidemiología local. La introducción
${ }^{1}$ Hospital Nacional de Itaugua Guazu- Ministerio de Salud Pública y Bienestar Social. Itaugua, Paraguay.

Los autores declaran no poseer conflicto de interés. Los fondos para la presente investigación provienen de fondos propios.

Recibido: 10 de agosto de 2017 Aceptado: 7 de julio de 2018

Correspondencia a: Gloria Celeste Samudio Domínguez

gsamudio.samudio@gmail.com 
de glicopéptidos como terapia empírica inicial, con todo lo que implica el uso generalizado de este antimicrobiano, es una práctica extendida en los diferentes centros ${ }^{17}$.

\section{Objetivo}

Conocer la etiología y el perfil microbiológico de los microorganismos aislados en niños con diagnóstico de sepsis neonatal tardía nosocomial internados en la terapia intensiva neonatal en un centro de alta complejidad.

\section{Material y Métodos}

Estudio observacional, retrospectivo, de prevalencia. Fueron incluidos al estudio todos los recién nacidos con diagnóstico de sospecha de sepsis nosocomial, independientemente de la edad gestacional y peso, internados en la Unidad de Cuidados Intensivos Neonatales (UCIN) con capacidad de 20 pacientes, de un hospital de tercer nivel, ubicado en área urbana, en el período de enero a diciembre de 2015.

Los datos clínicos y de laboratorio fueron recolectados de las fichas clínicas. Los datos bacteriológicos fueron tomados de las fichas clínicas y libros de microbiología.

Para el diagnóstico de infección del torrente sanguíneo, se consideró a los recién nacidos con signos, síntomas y/o laboratorio sugerente de infección bacteriana. Al establecerse la sospecha de infección nosocomial, todos los pacientes fueron sometidos a toma de cultivo de orina y hemocultivos periféricos para aerobios: dos o más muestras de sangre por punción de venas periféricas en sitios diferentes con técnica aséptica y se inocularon en frascos de hemocultivo para microorganismos aerobios.

Tabla 1. Microorganismos más frecuentemente aislados en los episodios de sepsis neonatal nosocomial ( $\mathrm{n}: \mathbf{8 8}$ )

\begin{tabular}{|c|c|c|c|c|c|c|c|c|}
\hline \multirow[t]{2}{*}{ Especie } & \multirow[t]{2}{*}{$\mathbf{n}$} & \multirow[t]{2}{*}{$\%$} & \multicolumn{2}{|c|}{ Sangre } & \multicolumn{2}{|c|}{ Orina } & \multicolumn{2}{|c|}{ S. traqueal } \\
\hline & & & $\mathbf{n}$ & $\%$ & $\mathbf{n}$ & $\%$ & $\mathbf{n}$ & $\%$ \\
\hline Staphylococcus epidermidis & 22 & 25 & 21 & 95,4 & 1 & 4,5 & 0 & 0 \\
\hline Acinetobacter baumannii & 10 & 11,3 & 3 & 30 & 0 & 0 & 7 & 70 \\
\hline Klebsiella pneumoniae & 8 & 9 & 1 & 12,5 & 6 & 75 & 1 & 12,5 \\
\hline Staphylococcus aureus & 6 & 6,8 & 6 & 100 & 0 & 0 & 0 & 0 \\
\hline Candida spp & 5 & 5,6 & 2 & 40 & 3 & 60 & 0 & 0 \\
\hline Enterococcus spp & 3 & 3,4 & 2 & 66,6 & 1 & 33,3 & 0 & 0 \\
\hline Serratia marcescens & 2 & 2,2 & 0 & 0 & 0 & 0 & 2 & 100 \\
\hline Elisabethkingia meningoseptica & 2 & 2,2 & 0 & 0 & 0 & 0 & 2 & 100 \\
\hline Escherichia coli & 1 & 1,1 & 0 & 0 & 1 & 100 & 0 & 0 \\
\hline Sin aislamiento & 29 & 33 & & & & & & \\
\hline Total & 88 & 100 & & & & & & \\
\hline
\end{tabular}

Los hemocultivos fueron considerados genuinamente positivos si existió crecimiento de bacterias gramnegativas o levaduras en uno o más frascos. Para especies grampositivas, se interpretaron como positivos si el aislamiento se había realizado en dos o más frascos; se consideró que el paciente presentaba infección si tenía datos clínicos y/o de laboratorio compatibles con un proceso infeccioso. Los urocultivos se tomaron con técnica aséptica, por cateterismo vesical. Se consideró positivo si contenía $>10^{5} \mathrm{ufc} / \mathrm{ml}$ de orina, con crecimiento de una única especie. Se consideró, a los efectos de este estudio, como infección de vías urinarias a la presencia de microrganismo en orina y alteraciones clínicas y/o de laboratorio compatibles con infección.

Se estudió 68 episodios de probable NAVM en 48 pacientes con sospecha de esta complicación, mediante cultivo de secreción traqueal (ST), según criterios establecidos para pacientes pediátricos menores de un año de edad, entre los que se incluyen fiebre, alteración de parámetros hematológicos, cambios radiológicos, cambios en las secreciones traqueales, además de aumento de parámetros respiratorios, criterios que deben presentarse al menos $48 \mathrm{~h}$ luego de la intubación o de la extubación ${ }^{18}$. Se consideró cultivo de ST positivo si el recuento de ufc era $\geq 10^{5}$ de un único microorganismo.

Los puntos de corte de las concentraciones inhibitorias mínimas (CIM) para definir resistencia o sensibilidad antimicrobiana fueron acordes a los criterios establecidos por el Clinical and Laboratory Standards Institute (CLSI).

Ninguno de los pacientes fue sometido a cirugía antes del episodio de infección nosocomial.

La unidad de análisis fue episodio de infección nosocomial.

\section{Resultados}

Fueron incluidos 70 recién nacidos (RN) los que presentaron 88 episodios de infección nosocomial, diagnosticados por manifestaciones clínicas y hallazgos de laboratorio.

La mayoría (89\%) de la población de $\mathrm{RN}$ con sepsis neonatal nosocomial fueron prematuros y con peso de nacimiento entre $<1.000$ y 1.500 g preferentemente (58\%).

Los pacientes fueron sometidos a diversos procedimientos invasores, por orden de frecuencia: uso de vías centralizadas $90 \%$, con una duración promedio de 11 días; asistencia respiratoria mecánica $76 \%$, con promedio de 7 días; cateterismo umbilical $67 \%$, con permanencia promedio de cuatro días; y nutrición parenteral $67 \%$, con promedio de duración de 7 días.

En los 88 episodios se aislaron 69 microorganismos en distintos tipos de muestras, siendo Staphylococcus epidermidis y Acinetobacter baumannii los más frecuentes (Tabla 1). 
El aislamiento a partir de hemocultivo periférico se obtuvo en 37 (40\%) de los casos. Los microorganismos más frecuentemente aislados a partir de sangre fueron del género Staphylococcus (Tabla 1). No fueron estudiadas en este reporte, las infecciones relacionadas a catéter venoso central.

De los 68 episodios de sospecha de NAVM estudiados, se confirmó en 43 (63\%), identificándose el agente responsable en $12(27 \%)$ de los casos. Las especies más frecuentemente aisladas en el aparato respiratorio fueron A. baumannii, Serratia marcescens y Elizabethkingia meningoseptica (Tabla 1).

En todos los pacientes se efectuó urocultivo obteniéndose aislado en 12 muestras de orina; se encontró más frecuentemente Klebsiella pneumoniae, seguido de Candida sp (Tabla 1).

Se realizó punción lumbar con examen de citoquímico y cultivo en 66 (75\%) de los casos; resultando patológico en 21 (32\%). Se recuperó un agente causal en sólo un caso, correspondiendo a Enterococcus casseliflavus.

\section{Susceptibilidad antimicrobiana in vitro}

Las características de estos episodios de infección nosocomial pueden visualizarse en la Tabla 2.

Tanto los aislados de Staphylococcus coagulasa negativa como Staphylococcus aureus fueron resistentes a meticilina. Los bacilos gramnegativos expresaron resistencia a cefalosporinas de tercera generación, productores de BLEE en $60 \%$, y en el caso de los aislados de $\mathrm{A}$. baumannii, expresaron sensibilidad sólo a colistina (Tabla 3).

La terapia empírica inicial consistió en meropenem unido a vancomicina y aminoglucósico, con ajuste de los antimicrobianos, según fuese el reporte microbiológico.

Del total de los 70 pacientes estudiados, 18 (26\%) presentaron un segundo episodio de infección nosocomial. La segunda infección nosocomial se observó sólo en la población de prematuros.

La mortalidad con el primer episodio de infección nosocomial fue de $34 \%$, aumentando a $50 \%$ las probabilidades de fallecer si hubo un segundo episodio en el paciente. No se encontró asociación entre la mortalidad ocasionada por el segundo episodio con el sexo, edad gestacional igual o menor a 33 semanas $(\mathrm{p}<0,01)$.

\section{Discusión}

La sepsis neonatal nosocomial (SNN) es una entidad causante de alta morbimortalidad, costos administrativos elevados y secuelas a largo plazo en los recién nacidos.

Los neonatos, principalmente los prematuros y aquellos de bajo peso, se encuentran sometidos a diversos procedimientos invasores, diagnósticos o terapéuticos, que rompen barreras de protección, exponiendo al infante a diversos microorganismos que pueden causar infecciones
Tabla 2. Características de los episodios de infección nosocomial, según edad gestacional, sexo, sitio de infección y mortalidad ( $\mathrm{n}: 88$ )

\begin{tabular}{|c|c|c|c|c|c|}
\hline \multirow[t]{2}{*}{ Característica } & \multicolumn{2}{|c|}{$\begin{array}{l}\text { Primer episodio } \\
\quad n=70\end{array}$} & \multicolumn{2}{|c|}{$\begin{array}{c}\text { Segundo episodio } \\
n=18\end{array}$} & \multirow[b]{2}{*}{$p$} \\
\hline & $n$ & $\%$ & $n$ & $\%$ & \\
\hline Femenino & 33 & 47 & 8 & 44 & $<0,01$ * \\
\hline Masculino & 37 & 53 & 10 & 56 & \\
\hline \multicolumn{6}{|l|}{ EG semanas } \\
\hline 26 a 29 & 2 & 3 & 2 & 11 & \\
\hline 30 a 33 & 37 & 53 & 10 & 56 & $<0,01$ * \\
\hline 34 a 36 & 24 & 34 & 6 & 33 & \\
\hline 37 y más & 7 & 10 & 0 & & \\
\hline \multicolumn{6}{|l|}{ IN más frecuente } \\
\hline SRM en HMC & 21 & 30 & 8 & 44 & \\
\hline Mortalidad & 24 & 34 & 9 & 50 & $<0,01^{*}$ \\
\hline
\end{tabular}

*Chi cuadrado. IN: infección nosocomial. EG: edad gestacional. SRM: Staphylococcus resistente a meticilina. HMC: Hemocultivo.

Tabla 3. Sensibilidad in vitro de las tres principales especies bacterianas aisladas

\begin{tabular}{llc}
\hline Germen & Antimicrobiano & Resistencia \% \\
Staphylococcus epidermidis & Oxacilina & 96 \\
(n: 22) & Rifampicina & 25 \\
& Ciprofloxacina & 75 \\
& Cotrimoxazol & 43 \\
& Amikacina & 64 \\
Acinetobacter baumannii & Ampicilina/sulbactam & 55 \\
(n: 10) & Ceftazidima & 55 \\
& Ciprofloxacina & 73 \\
& Gentamicina & 64 \\
& Imipenem & 100 \\
& Meropenem & 73 \\
Klebsiella pneumoniae & Piperacilina/tazobactam & 20 \\
(n: 8) & Imipenem & 10 \\
& Gentamicina & 60 \\
& Ciprofloxacina & 40
\end{tabular}

graves y amenazar su vida. La rotura de la barrera dérmica, aunada a un sistema inmunológico inmaduro favorecen la generación de infecciones bacterianas y fúngicas graves, además de complicaciones propias de la prematuridad, tales como la retinopatía, sangrado intraventricular o displasia broncopulmonar ${ }^{1-3,19}$.

Conocer las bacterias nosocomiales que causan las infecciones en este grupo etario es fundamental para cada institución pues varían de centro a centro, de hospital a hospital y de país en país ${ }^{10-16}$. Este conocimiento facilita la instauración temprana y oportuna de la terapia empí- 
rica adecuada. La comparación de estas epidemiologías permite analizar las terapias empíricas y resistencias in vitro, un intercambio de experiencias en el uso de antimicrobianos y en las intervenciones a ser aplicadas para frenar las resistencias.

En la región y en el país predominan los bacilos gramnegativos productores de BLEE y Staphylococcus resistentes a meticilina, principalmente Staphylococcus coagulasa negativa ${ }^{7,15}$. Además la morbimortalidad parece ligada al peso del recién nacido ${ }^{15}$ en un trabajo anterior realizado en nuestro país, hallazgo con el que coincidimos ya que en el presente estudio encontramos que la mayor prevalencia fue en prematuros y en los de 1.001 a 1.500 gramos. Este resultado es de esperarse por los factores mencionados más arriba, en este mismo apartado.

La mayor frecuencia de aislados del género Staphylococcus se debe probablemente al importante uso de procedimientos invasores que causan disrupción en la barrera cutánea. Este hallazgo coincide con la literatura médica actual, que encuentra un predominio importante de este agente causal ${ }^{10,20}$. Los porcentajes de aislados de Staphylococcus coagulasa negativa varían según los diferentes autores, pero todos parecen coincidir en que este es el agente más frecuentemente encontrado en casos de sepsis neonatal tardía ${ }^{7,12,15,21,22}$.

En orden de frecuencia de microorganismos aislados siguen A. baumannii y K. pneumoniae, obtenidos de sangre, orina y secreción traqueal. Si bien llama la atención la presencia de A. baumannii sin estar en curso un brote epidémico, no es éste el primer reporte que cita a esta bacteria como causante de infección nosocomial en neonatos ${ }^{7,15,16}$. La presencia de Acinetobacter sp en sala de terapia intensiva es indicativo de que las medidas de contención y control han sido insuficientes. Dicho agente se encuentra en las superficies de mobiliarios y, una vez allí instalado, su erradicación es difícil de lograr, pudiendo provocar brotes o casos esporádicos, por lo que la vigilancia de la epidemiología bacteriana en las UCIN es de suma importancia ${ }^{20}$. En nuestro caso, A. baumannii era multi-resistente y sólo sensible a colistina, fármaco al que respondió en forma adecuada.

No menos preocupante son las infecciones causadas por $K$. pneumoniae, de alta morbilidad y mortalidad. Si bien la literatura médica actual menciona su resistencia a carbapenémicos como un factor negativo en aumento ${ }^{23}$, la frecuencia de aislamiento de $K$. pneumoniae en nuestro estudio es muy escaso para ponderar esta resistencia siendo de suma trascendencia realizar una vigilancia nacional de infecciones por $K$. pneumoniae en recién nacidos en el Paraguay. Aún así, pudimos constatar que la presencia de cepas productoras de BLEE alcanzó a 60\%. Esta resistencia podría ser ocasionada por uso descontrolado de antimicrobianos haciendo que la epidemiología local esté variando hacia bacterias cada vez más resistentes.
Los sitios frecuentes de infección son, por orden de importancia: infección del tracto sanguíneo, seguido por NAVM con comprobación clínica y certificación bacteriológica y, por último, infección de vías urinarias, lo cual coincide con la literatura científica consultada ${ }^{7,10-12,15}$.

Las intervenciones tendientes a disminuir este tipo de infección en las terapias intensivas, a través de paquetes de prevención de infecciones nosocomiales, son una herramienta importante y que debe ser utilizada tempranamente ${ }^{24}$.

Los microrganismos aislados de secreción traqueal y orina fueron escasos, por lo que no pueden extraerse conclusiones de prevalencias y resistencias aplicables a la población general, aunque en ambas situaciones encontramos predominio de bacilos gramnegativos. Deben realizarse mayores esfuerzos de recuperación bacteriológica en ambas entidades clínicas.

Un dato llamativo es la afectación del SNC, evidenciado como alteración del LCR compatible con MBA en un tercio de los casos. Si bien no se recuperó un microorganismo en la gran mayoría de ellos, esto se debió, muy posiblemente, a que la inestabilidad hemodinámica del paciente impidió realizar estudio del LCR en etapas tempranas de la infección nosocomial y los pacientes ya se encontraban recibiendo antimicrobianos de amplio espectro por varios días. La alta prevalencia de este hallazgo, en comparación con otros autores ${ }^{15,25}$ debe alertar al médico tratante a buscar de manera intencionada el compromiso meníngeo, realizando la punción lumbar una vez que el estado hemodinámico y respiratorio del paciente lo permita, a fin de evitar la pérdida del diagnóstico de infección del SNC dentro del cuadro de sepsis ${ }^{26}$.

La terapia empírica inicial debe ser lo más asertiva posible, para asegurar la cobertura de los posibles agentes causales; en nuestro estudio fue un carbapenémico unido a vancomicina y aminoglucósido, en consideración a los perfiles de resistencia ya descritos, adecuando al antibiograma una vez identificado el microorganismo causal, tratando de descalar lo más rápido posible.

Esta terapia empírica variará de un centro a otro, e incluso en el mismo centro a lo largo del tiempo, por lo cual es importante realizar vigilancia de la susceptibilidad in vitro de los agentes causales de infecciones nosocomiales. La presencia de una endemia de especies altamente resistentes a los antimicrobianos debería resolverse mediante un programa de control de infecciones y de uso racional de antimicrobianos.

La mortalidad por sepsis nosocomial es elevada, y en nuestro estudio, la presencia de un segundo episodio de infección nosocomial resultó ser un signo de mal pronóstico, elevando la mortalidad hasta $50 \%$; aunque no se encontró diferencia estadísticamente significativa entre la posibilidad de fallecer por el primer o segundo episodio de infección nosocomial, se deben realizar esfuerzos a fin de 
evitar el segundo episodio de SNN ya que esto aumenta la morbilidad y los costos de internación.

\section{Debilidades del estudio}

Por su carácter retrospectivo, no se pudo efectuar una intervención para mejorar el rendimiento de los cultivos. No hubo estudio de anaerobios estrictos. La escasa cantidad de especies gramnegativas recuperadas impidió hacer inferencias de las resistencias en estos agentes.

\section{Resumen}

Introducción: La sepsis neonatal nosocomial (SNN) es una entidad frecuente en las unidades de cuidados intensivos, donde causa una gran morbimortalidad. La ubicación más frecuente es bacteriemia, seguido de neumonía asociada a ventilador mecánico y vía urinaria. Objetivo: Conocer la etiología y localización más fre- cuente de la infección en el SNN. Población, Material y Métodos: Estudio retrospectivo, de prevalencias de enero a diciembre de 2015, realizado en la Unidad de Cuidados Intensivos Neonatal de un hospital de alta complejidad. Fueron incluidos todos los neonatos. Resultados: Se incluyeron 70 pacientes, se analizaron 88 episodios de SNN. La localización más frecuente fue sangre $40 \%$ de los casos, seguido de orina y aspirado traqueal en $25 \%$ respectivamente. Los microorganismos más frecuentemente aislados fueron Staphylococcus de diferentes tipos, seguido de Acinetobacter baumannii multi-resistente. La afectación del SNC fue de 32\%. La mortalidad fue de $34 \%$, elevándose a $50 \%$ ante un segundo episodio de SNN. La terapia empírica de elección fue vancomicina y carbapenem, ajustándose a antibiograma. Conclusiones: La infección más frecuente fue la bacteremia, principalmente por Staphylococcus resistentes a meticilina. La afectación del SNC fue elevada, lo mismo que la mortalidad.

\section{Referencias bibliográficas}

1.- Softić I, Tahirović H, Di Ciommo V, Auriti C. Bacterial sepsis in neonates: Single centre study in a neonatal intensive care unit in Bosnia and Herzegovina. Acta Med Acad. 2017; 46 (1): 7-15. doi: 10.5644/ama2006-124.181.

2.- Ríos Valdéz V, Navia Bueno M P, Díaz Villegas M, Salazar Fuentes J. Factores de riesgo asociados a sepsis neonatal Rev. Bol. Ped 2005; 44 (2): 87-92. http://www.scielo. org.bo/scielo.php?script=sci_arttext\&pid $=$ S1024-06752005000200004.

3.- Kan B, Razzaghian H R, Lavoie P M. An immunological perspective on neonatal sepsis. Trends Mol Med 2016; 22 (4): 290-302. doi: 10.1016/j.molmed.2016.02.001.

4.- Rodríguez-Weber M A, López-Candiani C, Arredondo-García J L, Gutiérrez-Castrellón P, Sánchez-Arriaga F. Morbilidad y mortalidad por sepsis neonatal en un hospital de tercer nivel de atención. Salud Pública Méx 2003; 45 (2): 90-5. https://www.ncbi.nlm.nih.gov/ pubmed/12736986.

5.- Coria-Lorenzo J de J, Revilla Estivill N F, SotoRomero IE, Saavedra-Barrios M A, GadeaAlvarez T. Epidemiología de las infecciones nosocomiales neonatales, en un hospital de especialidades pediátricas de la Ciudad de México (revisión de 3 años). Perinatol Reprod Hum 2000; 14 (3): 151-9. http://www. medigraphic.com/pdfs/inper/ip-2000/ip003d. pdf.

6.- Wu I H, Tsai M H, Lai M Y, Hsu L F, Chiang M $\mathrm{C}$, Lien R, et al. Incidence, clinical features, and implications on outcomes of neonatal late-onset sepsis with concurrent infectious focus. BMC Infect Dis 2017; 3: 17 (1): 465. doi: 10.1186/ s12879-017-2574-7.
7.- Lona Reyes J C, Verdugo Robles M A, Pérez Ramírez R O, Pérez Molina J J, Ascencio Esparza E P, Benítez Vázquez E A. Etiología y patrones de resistencia antimicrobiana en sepsis neonatal temprana y tardía, en una Unidad de Terapia Intensiva Neonatal. Arch Argent Pediatr 2015; 113 (4): 317-23. doi: 10.5546/ aap.2015.317.

8.- Garner J S, Jarvis W R, Emori T G, Horan T C, Hughes J M. CDC definitions for nosocomial infections, 1988. Am J Infect Control 1988; 16: 128-40. https://www.ncbi.nlm.nih.gov/ pubmed/2841893.

9.- Hornik C P, Benjamin D K, Becker K C, Benjamin Jr. D K, Li J, Clark R H, et al. Use of the complete blood cell count in late-onset neonatal sepsis. Pediatr Infect Dis J 2012; 31 (8): 803-7. doi: 10.1097/ INF.0b013e31825691e4.

10.- Hammoud M S, Al-Taiar A, Thalib L, Al-Sweih N, Pathan S, Isaacs D. Incidence, aetiology and resistance of late-onset neonatal sepsis: a fiveyear prospective study. J Paediatr Child Health 2012; 48 (7): 604-9. doi: 10.1111/j.14401754.2012.02432.x.

11.- Osrin D, Vergnano S, Costello A. Serious bacterial infections in newborn infants in developing countries. Curr Opin Infect Dis 2004; 17 (3): 217-24. https://www.ncbi.nlm.nih. gov/pubmed/15166824.

12.- De Souza Rugolo L M, Bentlin M R, MussiPinhata M, de Almeida M F, Lopes J M, Marba $\mathrm{S}$ T, et al; Brazilian Network on Neonatal Research. Late-onset sepsis in very low birth weight infants: a Brazilian Neonatal Research Network Study. J Trop Pediatr 2014; 60 (6): 415-21. DOI: 10.1093/tropej/fmu038.

13.- Medina-Mejia M, Hernández-Ramos I, NandìLozano ME, Ávila-Figueroa C. Infecciones nosocomiales en una unidad de cuidados intensivos neonatales. Perinatol Reprod Hum 2000; 14: 143-50. http://maternoinfantil.org/ archivos/smi_D576.pdf.

14.- Samayam P, Ravi Chander B. Study of urinary tract infection and bacteriuria in neonatal sepsis. Indian J Pediatr 2012; 79 (8): 1033-6. doi: 10.1007/s12098-012-0727-7.

15.- Genes L, Lacarrubba J, Mir R, Céspedes E, Mendieta E. Neonatal sepsis in very-low birthweight newborns over an 11 year period. Pediatr (Asunción) 2013; 40 (2): 145-54 http://scielo. iics.una.py/pdf/ped/v40n2/v40n2a05.pdf.

16.- Labi A K, Obeng-Nkrumah N, Bjerrum S, Enweronu-Laryea C, Newman M J. Neonatal bloodstream infections in a Ghanaian Tertiary Hospital: Are the current antibiotic recommendations adequate? BMC Infect Dis 2016; 16 (1): 598. DOI: 10.1186/s12879-0161913-4.

17.- Różańska A, Wójkowska-Mach J, Adamski P, Borszewska-Kornacka M, Gulczyńska E, Nowiczewski M, et al. Antibiotic consumption in laboratory confirmed vs. non-confirmed bloodstream infections among very low birth weight neonates in Poland. Ann Clin Microbiol Antimicrob. 2017; 16 (1): 20. doi: 10.1186/ s12941-017-0196-y.

18.- Fica C A, Cifuentes D M, Hervé E B. Actualización del Consenso Neumonía Asociada a Ventilación Mecánica Primera parte. Aspectos diagnósticos. Rev Chil Infectol 2011; 28 (2): 130-51. http://dx.doi.org/10.4067/ S0716-10182011000200005.

19.- Leal Y A, Álvarez-Nemegyei J, Velázquez J R, Rosado-Quiab U, Diego-Rodríguez N, Paz-Baeza E, et al. Risk factors and prognosis for neonatal sepsis in southeastern Mexico: analysis of a four-year historic cohort follow- 
up. BMC Pregnancy Childbirth 2012; 12 (48): 3-9 doi: 10.1186/1471-2393-12-48.

20.- Lu Q, Zhou M, Tu Y, Yao Y, Yu J, Cheng S. Pathogen and antimicrobial resistance profiles of culture-proven neonatal sepsis in Southwest China, 1990-2014. J Paediatr Child Health 2016; 52 (10): 939-43. doi: 10.1111/jpc. 13278. Epub 2016 Aug 8.

21.- Kohli-Kochhar R, Omuse G, Revathi G. A tenyear review of neonatal bloodstream infections in a tertiary private hospital in Kenya. J Infect Dev Ctries 2011; 5 (11): 799-803 https://www. ncbi.nlm.nih.gov/pubmed/?term=Kohli-Kochha $\mathrm{r}+\mathrm{R} 1 \% 2 \mathrm{C}+$ Omuse $+\mathrm{G} \% 2 \mathrm{C}+$ Revathi $+\mathrm{G}$.

22.- Mugalu J, Nakakeeto M K, Kiguli S, Kaddu-
Mulindwa D H. Aetiology, risk factors and immediate outcome of bacteriologically confirmed neonatal septicaemia in Mulago hospital, Uganda. Afr Health Sci 2006; 6 (2): 120-6. https://www.ncbi.nlm.nih.gov/pmc/ articles/PMC1831983/

23.- Yusef D, Shalakhti T, Awad S, Algharaibeh $\mathrm{H}$, Khasawneh W. Clinical characteristics and epidemiology of sepsis in the neonatal intensive care unit in the era of multi-drug resistant organisms: A retrospective review. Pediatr Neonatol 2018; 59 (1): 35-41 doi: 10.1016/j. pedneo.2017.06.001.

24.- Tanguy M, Kouatchet A, Tanguy B, Pichard É, Fanello S, Joly-Guillou M L. Management of an Acinetobacter baumannii outbreak in an intensive care unit. Med Mal Infect 2017; 19. S0399-077X(17)30684-4. doi: 10.1016/j. medmal.2017.06.003.

25.- McMullan R, Gordon A. Impact of a central line infection prevention bundle in newborn infants. Infect Control Hosp Epidemiol 2016; 37 (9): 1029-36. doi: 10.1017/ice.2016.127.

26.- Zea-Vera A, Turín C G, Rueda M S, GuillénPinto D, Medina-Alva P, Tori A, et al. Use of lumbar puncture in the evaluation of lateonset sepsis in low birth weight neonates. Rev Peru Med Exp Salud Pública 2016; 33 (2): 278-82. https://doi.org/10.17843/ rpmesp.2016.332.2215. 